\title{
Active Queue Management Mechanisms and its Effects on Voice over IP
}

\author{
Makkawi Abdelsalam Mohamedkheir'1, Osama Ali Abdelgadir², Yassin Abdulkarim Hamdalla ${ }^{3}$, \\ Fatima Abdelghani Mustafa ${ }^{4}$
}

\author{
${ }^{1}$ Future University, Department of Computer Engineering, Faculty of Engineering, Africa Avenue, Khartoum, Sudan \\ ${ }^{2}$ Future University, Department of Electronics \& Communication Engineering, Faculty Telecommunications \& Space Technology, Africa \\ Avenue, Khartoum, Sudan \\ ${ }^{3}$ Future University, Department of Electronics \& Communication Engineering, Faculty Telecommunications \& Space Technology, Africa
Avenue, Khartoum, Sudan
}

${ }^{4}$ Future University, Department of Computer Engineering, Faculty of Engineering, Africa Avenue, Khartoum, Sudan

\begin{abstract}
Due to the increase in the data been transmitted every single second around the networks all over the world, also the integration of both data and voice over the existing network infrastructure which ensure low cost, and enhance the network management which result of a huge amount of data crossing the links from this point come the need of queue management to control this data from the source to the destination, there been various development in this area, so two classifications was founded which is passive queue management (PQM) and active queue management, this paper focus in active queue management and its variant, basically this thesis presents an inclusive study about the effect of active queue management (AQM) on Voice over Internet Protocol (VoIP) quality of service Using simulations. The valuation is done using the OPNET Modeler, which offers a appropriate and easy-touse plat form for simulating all types of networks and this also give option to go through different levels of designing a network even with the ability to program the mechanism you want which is used here to implement two types of AQM mechanism which is not involved by default in the OPNET and these two mechanisms are ARED and GRED. The performance parameters used in the study are queue size, packet drop probability, and packet loss rate. The study shows that, among the four algorithms, ARED and GRED are more effective in reducing end-to-end delay and stabilizing the queue size which automatically reduce delay and jitter. In addition to that, it showed a significant impact on controlling the packet loss rate while maintaining high link utilization.
\end{abstract}

Keywords: AQM, ARED, GRED, WRED, JITTER and OPNET

\section{Introduction}

A clear increase in the use of computer network and internet according to the increases of the social applications and social network which is leads to point the attention to a very important topic which is quality of service QoS because the demand of users is increase, everyone need a reliable and very fast link to communicate with others, but there are a new problem appears when congestion occurs because of the oscillate of users from one node to another in the network in a matter of seconds which produce congestion that needed to be controlled, the main reason for congestion is that there are too many source sending too much data too fast for network to handle.

A lot of investigation is made in this serious issue and many researches was made to solve such crucial problem, and this effort bring out different approaches for dealing with this problem such as PQM passive queue management and AQM active queue management, example of PQM is drop tail which is the default method used in most routers in the network. And the wide spread researches is focused in AQM because of the diversity of the methods and algorithms used, Such as RED, ARED, GRED and WRED which we used here to investigate the effect of using any one of this method and see how it can deal with special types of data which is voice that transmitted in the same links with the data which named VOIP, voice over internet protocols.
Difficulty of implement this mechanism in a real router and test the effect of any of them is the reason behind using simulation in this research so we use one of the powerful simulation tools which is Opnet Optimized network (OPNET) also the important of simulation cannot denied because simulation is the basic for testing and learning about the real system .

\section{VoIP}

Mobile As the popularity of the data services increases but still voice is the most important service that remain the biggest earning source for the network service providers .There are two way used to deliver voice services, the first and the old one which is the Traditional voice networks utilized circuit-switching technologies, effectively a pair of copper wires is used to connect one party to the other, completing an electrical "circuit". and the other one is packet switching networks, With packet-switching, traditionally used by IP based networks such as the internet to transmit data, voice signals are broken down into tiny "packets" of digital sampled data, sent over the network and then reassembled at the receiving end. The efficiency in voice over IP is in part down to this packet process. Multiple conversations (as many as 6,000) can be transmitted over one pair of copper wires. VoIP stands for voice over internet protocol, unlike the traditional circuit-committed protocols of the public switched telephone network (PSTN), in VoIP 


\section{International Journal of Science and Research (IJSR) \\ ISSN (Online): 2319-7064}

Index Copernicus Value (2013): 6.14 | Impact Factor (2013): 4.438

voice signal is compressed and converted to digital voice packets, VoIP then uses the Internet Protocol (IP) for managing voice packets over IP network. Therefore, VoIP can be deployed on any IP enabled data network, such as the Internet, Ethernet, fabric or wireless network .

Due to the explosion of broadband in recent years, more and more people have fast internet connections at their disposal. With VoIP, every call you make can be treated as a "local" call - the internet has no concept of international. When you access a web page in the United States from the United Kingdom, you don't pay an "international" rate - you simply have a flat rate connection fee.

VoIP can be viewed as a similar utility to web access or email - if you're on the internet with a fast enough connection, you can speak to another party without incurring any call charges whatsoever, as long as the other party is using a device compatible with yours and this is one of the reasons for the increasing of the amount of VIOP uses over the networks.

Packet forwarding latency can have a large impact on the user experience for a variety of network applications. The applications most commonly considered as latency-sensitive are real-time interactive applications such as VoIP, video conferencing and networked "twitch" games .There are established models for the degradation in user experience for VoIP caused by latency

Every piece of network equipment has to have some amount of buffering in order to handle bursts of packets on an ingress link, and then to play them out on an egress link. It's particularly important in cases where there is a mismatch between the rate into the device and the rate out. For example, imagine a switch with a GigE ingress link and a 100 Mbps egress link. Even if the average ingress rate is 100 Mbps, the ingress link will often provide that traffic in bursts of packets at 1 Gbps. Buffering is pretty important to make sure that the switch can accept those bursts and play them out on the 100 Mbps link. From the perspective of egress link utilization, more buffering is better, since it reduces the chance that the egress link will go idle. For bulk TCP traffic (file transfers), user experience is driven by how quickly the file transfer can complete, which is directly related to how effectively the protocol can utilize the network links, again supporting the view that more buffering is better. But the downside to large buffers is that they result in excessive latency. While this isn't an issue for the bulk file transfers, it is clearly an issue for other traffic, and the issue is exacerbated by the TCP itself. The majority of TCP implementations use loss-based congestion control, which means the TCP ramps up its congestion window (effectively ramps up its sending rate) until it sees packet loss, then it cuts its congestion window in half, and then starts ramping back up again until it sees the next packet loss, and that saw-tooth continues. In a lot of networks, especially wired networks, packet loss doesn't come from noise on the wire. It comes from buffers being full, and when a packet arrives at a full buffer it has to be discarded. This is how the TCP automatically adjusts its transmission rate to match the available capacity of the bottleneck link. and this will be very sensitive for VIOP packets.

\section{AQM}

Bandwidth becomes the scarcest resource in the networks, and available bandwidth for services is even lower, as the maintenance of the network requires a part of the bandwidth, in addition to that asymmetric bandwidth capabilities are common in the internet [3] this causes that asymmetric bandwidth availability, differing down-link and up-link capabilities. Actually the out link provides less bandwidth than the incoming link. This leads to some situation when Active Queue Management (AQM) is intended to achieve high link utilization with a low queuing delay. Recent studies show that RED, one of the most well-known AQMs, is difficult to configure and does not provide significant performance gains given the complexity required for proper configuration. Recent variants of RED, such as AdaptiveRED are designed to provide more robust RED performance under a wider-range of traffic conditions but have not yet been evaluated. Active Queue Management (AQM) proposes to replace drop-tail queue management in order to improve network performance in terms of delay, link utilization, and packet loss rate and system fairness. AQM enhances routers to detect and notify end-systems of impending congestion earlier, allowing responsive traffic sources to reduce their transmission rates before congested router queues overflows. AQM has many types as we mentioned above so the next section will describe each one and its properties.

\subsection{RED Family AQM Mechanisms}

Random Early Detection (RED) algorithm [6] is a congestion avoidance technique highly used in modern communication networks for avoiding network congestion. Compared to existing algorithms, the RED system monitors network traffic loads in an effort to anticipate and avoid congestion at common network bottlenecks i.e. the system triggers before any congestion actually occurs. Moreover, RED uses AQM techniques currently deployed in large IP networks. It takes advantage of $\mathrm{TCP}^{\mathrm{ee}} \mathrm{s}$ congestion control mechanism to avoid congestion in network where packets are dropped probabilistically prior to periods of high congestion. RED monitors impending congestion by keeping an exponential weighted moving average of the queue (q). When congestion is detected, indicated by the queue average rising above a fixed minimum threshold ( ), incoming packets are randomly marked (drop or ECN) with a probability. The probability of the mark increases linearly from zero at to a maximum mark probability( ) at the maximum threshold When the queue average rises above RED drops all incoming packets to ensure a low average queuing delay. RED supports ECN marking while the queue average is in between the and the We will call RED using ECN marking RED-ECN hereafter. The performance benefits of RED can be maximized when RED uses ECN marking and is "well-configured" under the traffic load, in which the queue average does not significantly oscillate and stays under Unfortunately, studies show that RED is hard to configure for a wide-range of traffic mixes and loads . 


\section{International Journal of Science and Research (IJSR) \\ ISSN (Online): 2319-7064 \\ Index Copernicus Value (2013): 6.14 $\mid$ Impact Factor (2013): 4.438}

\subsubsection{RED Functionality}

Using The RED algorithm involves in computing the average queue size and compares the two thresholds, a minimum and a maximum one. If the average queue size is below the minimum threshold, the packet is not dropped and is sent to the buffer. On the other hand, when the average queue size is above the maximum threshold, each arriving packet is dropped. When the average queue size is between the minimum and maximum thresholds then the arriving packet is marked with a packet drop probability pa.

Since this probability is a function of the average queue size, every time a packet is marked with it, then the probability that a packet is marked from a particular connection is roughly proportional to that connection se share of the bandwidth at the router. Moreover this probability determines whether the packet is dropped or not as shown in figure 2.6.The minimum threshold value should be set high enough to maximize the link utilization. If the minimum threshold is too low, packets may be dropped unnecessarily, and the transmission link will not be fully used.

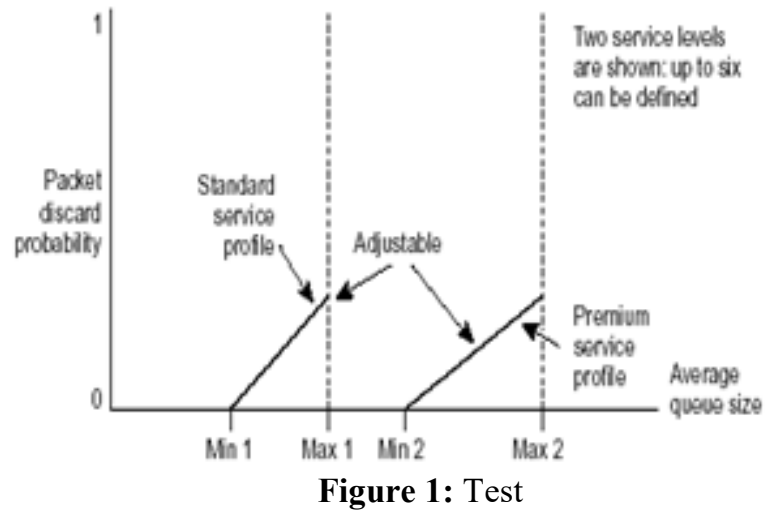

\subsubsection{RED's impact on traffic flow}

Usually, UDP based application can be considered as smooth traffic. At the end host TCP congestion control mechanism is implemented. TCP should respond to packet droppings after a RTT. The upper layer application take care of congestion and further retransmission whereas UDP hosts neglect packet loss and keep pumping data into network. It does not 14 mean that RED algorithm has no impact on UDP application. It has impact on all links of internet traffic, including both TCP and UDP connections.

The RED algorithm detects congestion and measures the traffic load level in the queue using the average queue size avg [12]. This is calculated using an exponentially weighted moving average filter and can be expressed as

$$
a v g=(1-w q) * a v g+w q * q,
$$

large numbers of mobile devices, it is not practical to configure keys between a mobile device and every other correspondent node. In the case of triangle routing, it"s conceivable to configure a key between mobile device and its home agent.

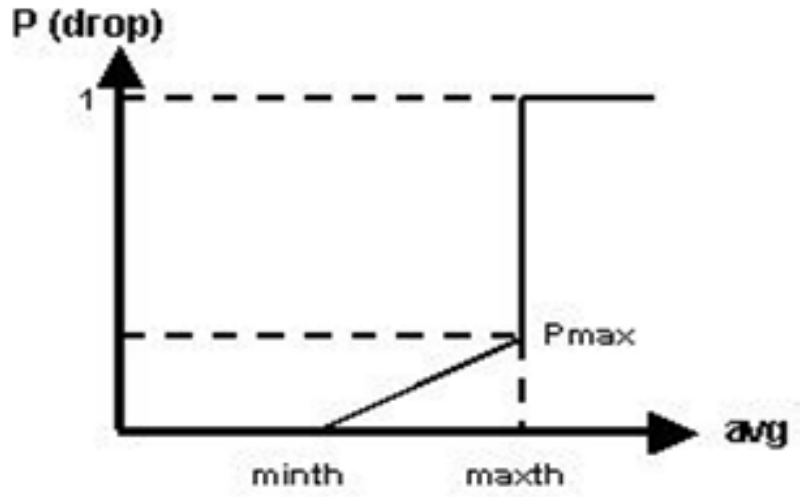

Figure 2.3: The RED mechanism.

Table 3.1: RED parameters

\begin{tabular}{|c|c|}
\hline AQM classification & Random Early Detection \\
\hline Goal of algorithm & $\begin{array}{c}\text { provide performance guarantee } \\
\text { (delay, throughput) }\end{array}$ \\
\hline Solution approach & Heuristic \\
\hline Congestion detection & $\begin{array}{c}\text { Qavg weighted average with burst- } \\
\text { awareness }\end{array}$ \\
\hline Fair bandwidth allo & $\begin{array}{c}\text { No, all flows have the same } \\
\text { dropping probability }\end{array}$ \\
\hline Malicious-aware & No, as not fair \\
\hline Target quality interval & $\begin{array}{c}\text { Qmin }<=\text { Qcurr }<=\text { Qmax. Where } \\
\text { Qmin and Qmax are static }\end{array}$ \\
\hline Required state & $\begin{array}{c}\text { O(1). Maintaining Qavg, but no } \\
\text { history }\end{array}$ \\
\hline \#of predef.pararms & 4 \\
\hline What has effect on Pdro & Qcurr,Qmin,Qmax. Pmax. Wq \\
\hline Special characteristic & $\begin{array}{c}\text { Reference AQM algorithm , burst- } \\
\text { awareness adjustable }\end{array}$ \\
\hline
\end{tabular}

\subsection{GRED: Gentle Random Early Detection}

GRED was proposed as an AQM method that controls congestion at router buffers preliminary GRED aims to solve some of RED's problems [13, 14] Gentle Red (GRED,[9]) is a variant of the RED algorithm. The discarding function of GRED is different. Going from maxp to 1 is not an abrupt change; a linear function with slope (1 maxp)/maxth is followed until $\mathrm{t}=2$ maxth is reached.

Table 3.2: GRED parameters

\begin{tabular}{|c|c|}
\hline AQM classification & Gentle Random Early Detection \\
\hline Goal of algorithm & $\begin{array}{c}\text { Adaptive trade-off between link } \\
\text { utilization and delay }\end{array}$ \\
\hline Solution approach & Heuristic \\
\hline Congestion detection & $\begin{array}{l}\text { Qavg, weighted average with } \\
\text { burst awareness }\end{array}$ \\
\hline Fair bandwidth allo & No \\
\hline Malicious-aware & No, as not fair \\
\hline Target quality interval & Static target interval Q target \\
\hline Required state & $\mathrm{O}(1)$ \\
\hline \#of predef.pararms & 3 \\
\hline What has effect on Pdro & $\begin{array}{l}\text { Qcurr, Qmin, Qmax , Qtarg, } \\
\text { doable Qmax t=2maxth }\end{array}$ \\
\hline Special characteristic & $\begin{array}{l}\text { Adapt Qtarg to meet delay and } \\
\text { throughput requirement }\end{array}$ \\
\hline
\end{tabular}




\section{International Journal of Science and Research (IJSR) \\ ISSN (Online): 2319-7064 \\ Index Copernicus Value (2013): 6.14 | Impact Factor (2013): 4.438}

\subsection{ARED: Adaptive Random Early Detection}

The adaptive RED or active RED (ARED) algorithm infers whether to make RED more or less aggressive based on the observation of the average queue length. If the average queue length oscillates around min threshold then early detection is too aggressive. On the other hand if the average queue length oscillates around max threshold then early detection is being too conservative. The algorithm changes the probability according to how aggressively it senses it has been discarding traffic [11]. Adaptive RED successfully "auto-tunes" the various RED parameters to achieve reliably good results.

The overall guidelines for Adaptive RED as implemented here are the same as those for the original Adaptive RED from [28], that is, of adapting maxp to keep the average queue size between min threshold and max threshold this approach differs from original Adaptive RED in four ways:

- maxp is adapted not just to keep the average queue size between min threshold and max threshold but to keep the average queue size within a target range half way between min threshold and max threshold

- Maxp is adapted slowly, over time scales greater than a typical round-trip time, and in small steps.

- Maxp is constrained to remain within the range [0.01, 0.5] (or equivalently, $[1 \%, 50 \%]$ ).

- Instead of multiplicatively increasing and decreasing maxp we use an additive-increase multiplicative decrease (AIMD) policy [15]

Table (3.3) ARED parameters

\begin{tabular}{|c|c|}
\hline AQM classification & Adaptive Random Early Detection \\
\hline Goal of algorithm & $\begin{array}{c}\text { Adaptive trade-off between link } \\
\text { utilization and delay }\end{array}$ \\
\hline Solution approach & Heuristic \\
\hline Congestion detection & $\begin{array}{c}\text { Qavg, weighted average with burst } \\
\text { awareness }\end{array}$ \\
\hline Fair bandwidth allo & No \\
\hline Malicious-aware & No, as not fair \\
\hline Target quality interval & Static target interval Qtarget \\
\hline Required state & 3 \\
\hline \#of predef.pararms & Qcurr,Qmin,Qmax,Qtarg $\alpha, \beta$, \\
\hline What has effect on Pdro & $\begin{array}{c}\text { Adapt Qtarg to meet delay and } \\
\text { throughput requirement }\end{array}$ \\
\hline Special characteristic &
\end{tabular}

\subsection{WRED: Weighted Random Early Detection}

Queues are provided to buffer traffic levels that exceed the bandwidth of individual ports. For each output port, a set of eight priority queues is allocated on each inbound traffic manager. When traffic exceeds the bandwidth of a port, packets are dropped randomly as long as the congestion persists. Under these conditions, traffic of greater priority can be dropped instead of traffic with a lesser priority.

Instead of being subject to this random process, you can configure a Brocade device to monitor traffic congestion and drop packets according to a WRED (Weighted Random Early Discard) algorithm. This algorithm enables the system to detect the onset of congestion and take corrective action.
In practice, WRED causes a device to start dropping packets as traffic in the device starts to back up. WRED provides various control points that can be configured to change a system's reaction to congestion. The following variables are used when calculating whether to drop or forward packets:

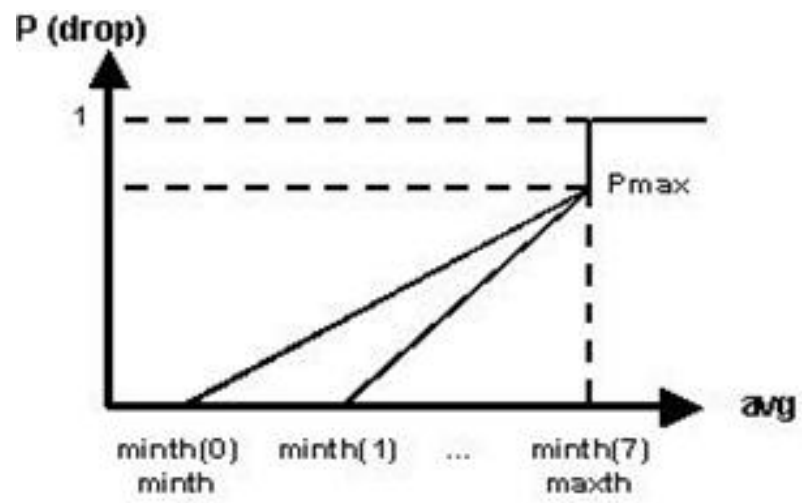

Figure 3.4: The WRED mechanism

The WRED algorithm that we want to use is different than the one implemented in the OPNET Tool. The OPNET Tool has different minimum average queue size for each packet with different ToS Byte, but the same maximum average queue size and dropping probability (Pmax). This case is presented in Figure 2.4. The minth( 0$)$,

minth(1) ... minth(7) are the minimum average queue size for the packet with ToS Byte $0,1, \ldots, 7$ respectively. The function that computes the minth, is given below: $\min t h($ ToS $)=\min t h+(\max t h-\min t h) *$ ToS $/ 7$

Table 2.4: WRED parameters

\begin{tabular}{|c|c|}
\hline AQM classification & Weighted Random Early Detection \\
\hline Goal of algorithm & $\begin{array}{c}\text { Classify the traffic according to the IP } \\
\text { precence }\end{array}$ \\
\hline Solution approach & Heuristic \\
\hline Congestion detection & $\begin{array}{c}\text { Qavg weighted average with burst- } \\
\text { awareness }\end{array}$ \\
\hline Fair bandwidth allo & $\begin{array}{c}\text { No, all flows have the same dropping } \\
\text { probability }\end{array}$ \\
\hline Malicious-aware & No, as not fair \\
\hline Target quality interval & $\begin{array}{c}\text { Qmin }<=\text { Qcurr }<=\text { Qmax. Where Qmin and } \\
\text { Qmax are static }\end{array}$ \\
\hline Required state & O(1). Maintaining Qavg, but no history \\
\hline \#of predef.pararms & 4 \\
\hline $\begin{array}{c}\text { What has effect on } \\
\text { Pdro }\end{array}$ & $\begin{array}{c}\text { Qcurr,Qmin,Qmax. Pmax. Wq } \\
\text { Special characteristic }\end{array}$ \\
\hline $\begin{array}{c}\text { Reference AQM algorithm, burst- } \\
\text { awareness adjustable }\end{array}$ \\
\hline
\end{tabular}

\section{Quality of Service}

Quality of service (QoS) is a network term that specifies a guaranteed throughput level. In layman's terms, it means that the network must be designed so that the voice and video data are transmitted through the network with a minimum of delay and loss. The network must be carefully evaluated to insure that it will be able to transmit voice and video data properly. Often components in the network must be upgraded or additional routers, switches, or packet shaping" devices may be required [16]. 


\section{International Journal of Science and Research (IJSR) \\ ISSN (Online): 2319-7064 \\ Index Copernicus Value (2013): 6.14 | Impact Factor (2013): 4.438}

QoS is a concept that characterizes the performance and operation of a communication end-to-end between two end points on a network. Another way of explaining this concept would be as the ability of a network element (application, host or router) to provide some level of assurance that the service requirements are met. In Internet key technology provides the best effort service called. Generally whenever information in the network is required, it has to go through several networks, therefore, the quality of end-to-end service depends mainly on the characteristics of QoS of the links and routers that must cross its path. Therefore, several tools are essential to achieve the QoS required by the type of application and user. To support QoS end-to-end is important to know more about the behavior dynamic networks. This is possible to do this by some parameters that can be measured and monitored on the network. Among the most important parameters are: width band, delay, jitter and packet loss probability.

\section{Simulation Setup}

The simulation was setup using the OPNET network simulator. The simulation environment consists of network components (VIOP devices) communicating with number of switches connected with routers forming the bottleneck topology .OPNET (Optimized Network Engineering Tool) is a simulation tools provides a virtual network environment for the specification, simulation and performance analysis of communication networks. OPNET Modeler uses different modeling levels and paradigms to represent the different network components. Each level is associated with a domain, and an editor. To make the development more intuitive to the user, the editors are hierarchically organized, so that the models developed in the Project Editor (Project Editor) depend on elements developed in the Node Editor (Node Editor). This in turn uses models defined Editor Processes (Process Editor). These are the three main editors OPNET, but there are also are complementary and Link Model Editor (to create, edit and view models link) Packet Format Editor (used to develop a particular packet format) or Probe Editor (Configuration of the statistics to be obtained during simulation).for all this reason we choose to design this study by this simulation tools. Both routers are connected to each other via the PPP DS1 cable. The PPP DS1 connects two systems running IP and it has a data rate of $1.544 \mathrm{Mbps}$. Other devices are connected to the routers with the 10 Base T cables which transmit data at $10 \mathrm{Mbps}$ and have a maximum length of 100 meters. The model includes an application definition, a QoS definition and a problem definition. The application definition consists of a list of the applications to be run on the network such as VoIP applications, FTP applications, and video applications. The applications send traffic into the network which are tested and analyzed. The QoS definition characterizes protocols that are supported at the IP layer. It defines different scheduling algorithms such as FIFO, WFQ, Custom algorithm and PQ. The pro le configuration is used to create user profiles. It can be specified on different network interfaces to create traffic on the application layer. Since this simulation focuses on Quality of Service in VoIP, hence the QoS profile has been included in the simulation and different scheduling algorithms have been set for the study.The experiments are based on G.711 encoder. The G.711 encoder is based on uncompressed Pulse Code Modulation (PCM) voice and could be used to encode straight from a traditional telephone network, The G.711 use 8 bits per sample and each sample is generated every 125 microseconds with the use of PCM and this leads to a bit rate of $64 \mathrm{Kbps}$ and is ideal for VoIP because of its low bandwidth. The simulation scenario tested on a dell laptop inspiron N5110 hardware platform with a computer name CTS running under windows 8 pro operating system with Intel(R) core ${ }^{\mathrm{TM}}$ i5-2410M CPU @ 2.30GHz for the processor and 4.00GB of RAM.

\subsection{Modelling Assumptions}

- The network operates at $100 \mathrm{Mb} / \mathrm{s}$ throughout the simulation except for the link between routers which will be much lower (E1) to form the congestion situations.

- There is no other network traffic besides VOIP traffic in this study.

- The simulated scenarios were going to investigate impact of different AQM mechanism changing the numbers of VOIP clients and some important parameters like maximum queue size.

\subsection{Topology Description}

A typical topology used in this project is in the form of "bottleneck", with five users and one Ethernet server, two switches and two routers. The capacity of the link between the two routers will be much lower compared to the other links, to create the situation of congestion control and experience packet drop and other effects. Three other important configuration parameters were used: application, profile and QoS profiles.

\section{Simulation parameters}

\subsection{Simulation Parameters for first scenario}

The below table shows the simulation parameters that used in this scenario, this scenario based on changing the number of users while the other parameters remain constant

Table 6.1: Simulation parameters

\begin{tabular}{|c|c|}
\hline Number of User & Variable from 5-300 \\
\hline Data forwarding rate (packets/second) & 500000 \\
\hline Maximum queue size & 100 \\
\hline Minimum threshold & 10 \\
\hline Maximum threshold & 20 \\
\hline Mark probability denominator & 0.1 \\
\hline Simulation duration & 1 hour \\
\hline Encoder scheme & G.711 (silence) \\
\hline Voice frame per packet & 5 \\
\hline
\end{tabular}

\subsubsection{Results}

\subsubsection{First Scenario : numbers of users is 5}


International Journal of Science and Research (IJSR)

ISSN (Online): 2319-7064

Index Copernicus Value (2013): 6.14 $\mid$ Impact Factor (2013): 4.438

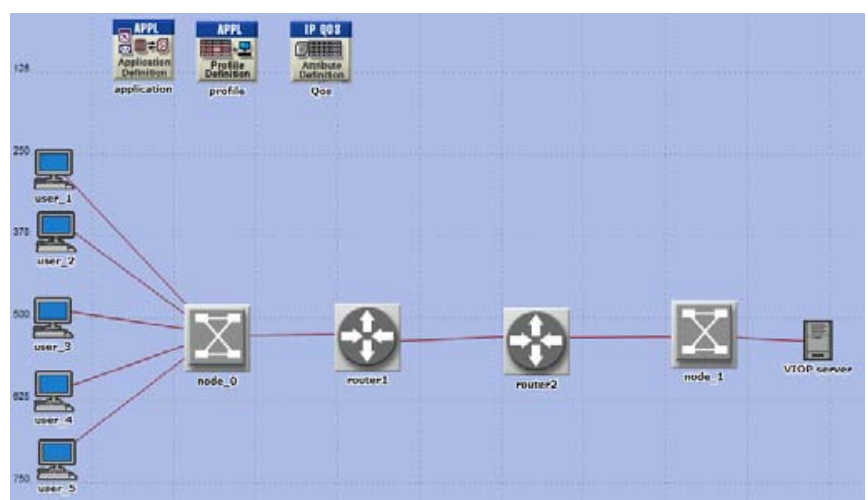

Figure 6.4: 5 users Network topology using OPNET

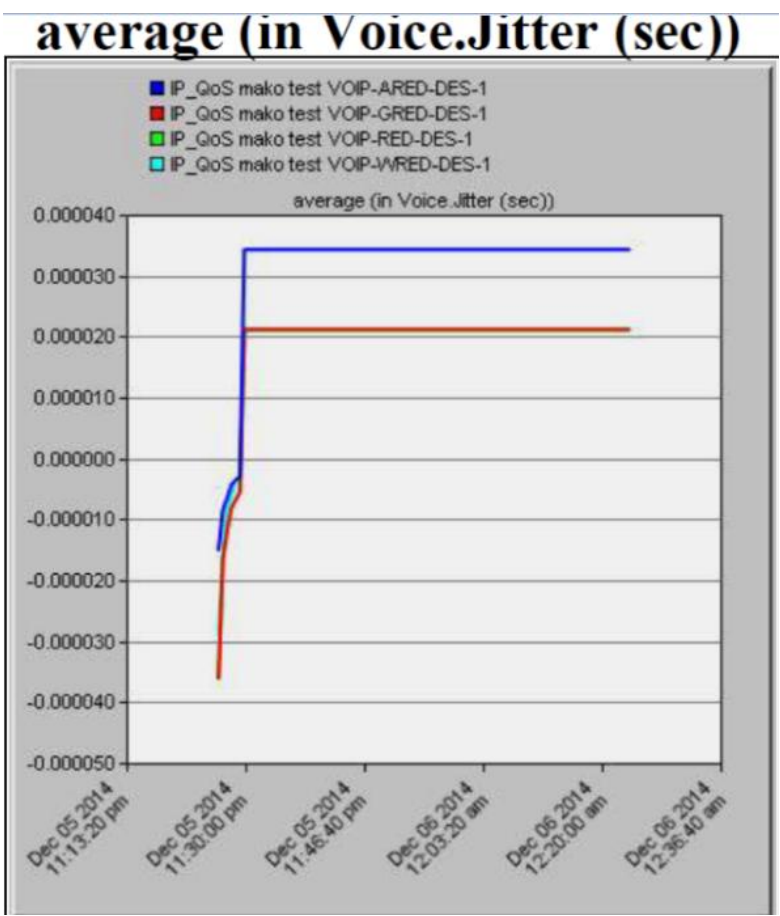

Figure 6.5: shows the graphic result of average jitter average (in Voice.Packet End-to-End Delay (sec))

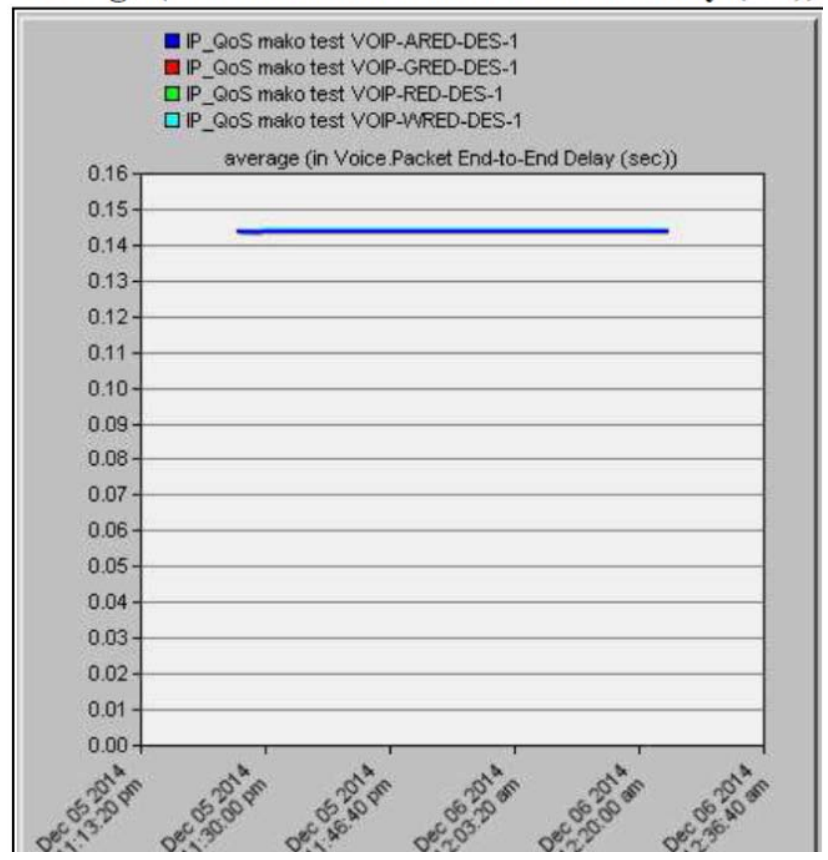

Figure 6.6: shows the graphic result of voice packet $E$ to $E$ delay router $A$ <-> router $B$ [0] of FIFO net <--

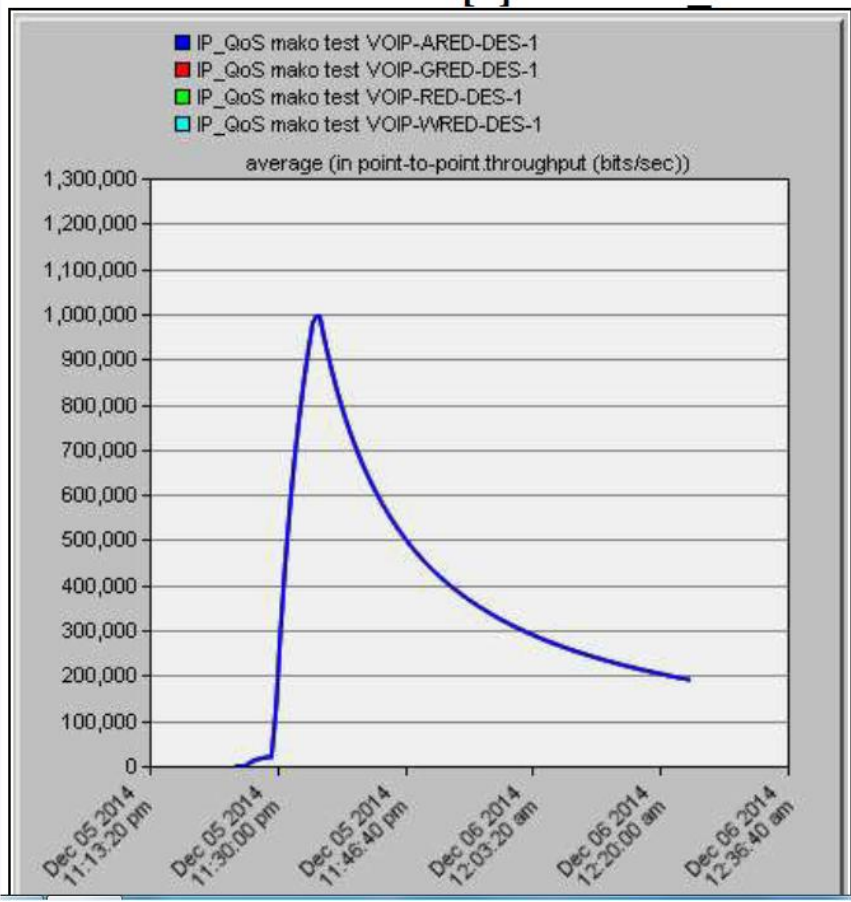

Figure 6.7: shows the graphic result Router A to B FIFO

\section{router $A$ of FIFO net}

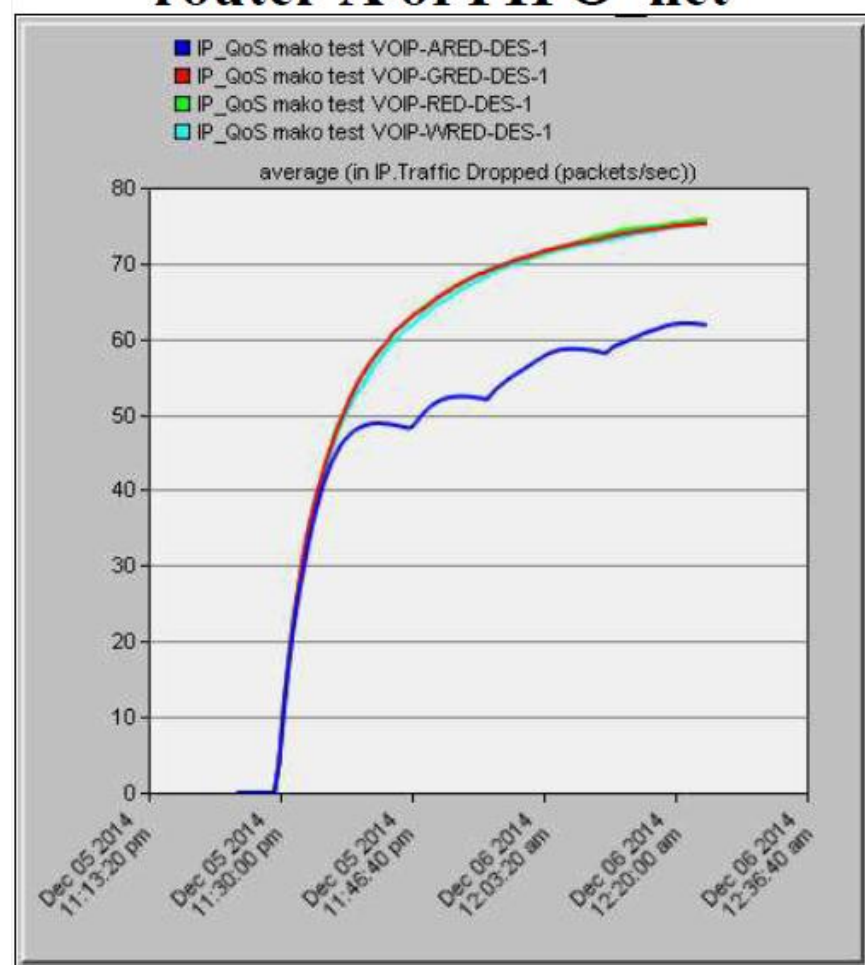

Figure 6.8: shows the graphic result Router A FIFO

The figures above describe the performance of the fourth AQM mechanism that been used in this study, it descrbes the performance when the number of users is 5 , the result shows that the GRED is the best mechanism reducing the jitter and the othe mechanism fall almost in the same level, end -to end delay is approximatly equal for all the mechanism that been used, in figure (d) ARED show best result of packet been droped, and other mechanism have a slight 


\section{International Journal of Science and Research (IJSR) \\ ISSN (Online): 2319-7064}

Index Copernicus Value (2013): 6.14 | Impact Factor (2013): 4.438

6.1.1.2 Second Scenario : numbers of users is 300

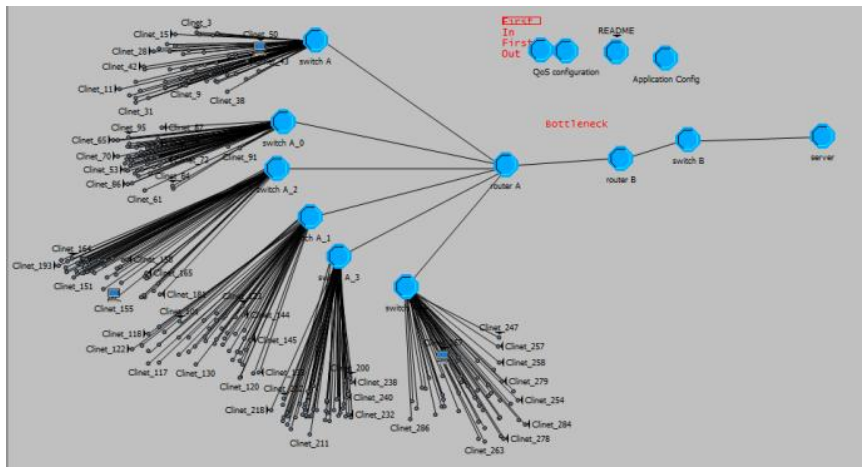

Figure 6.9: 300 users Network topology using OPNET

\section{average (in Voice.Jitter (sec))}

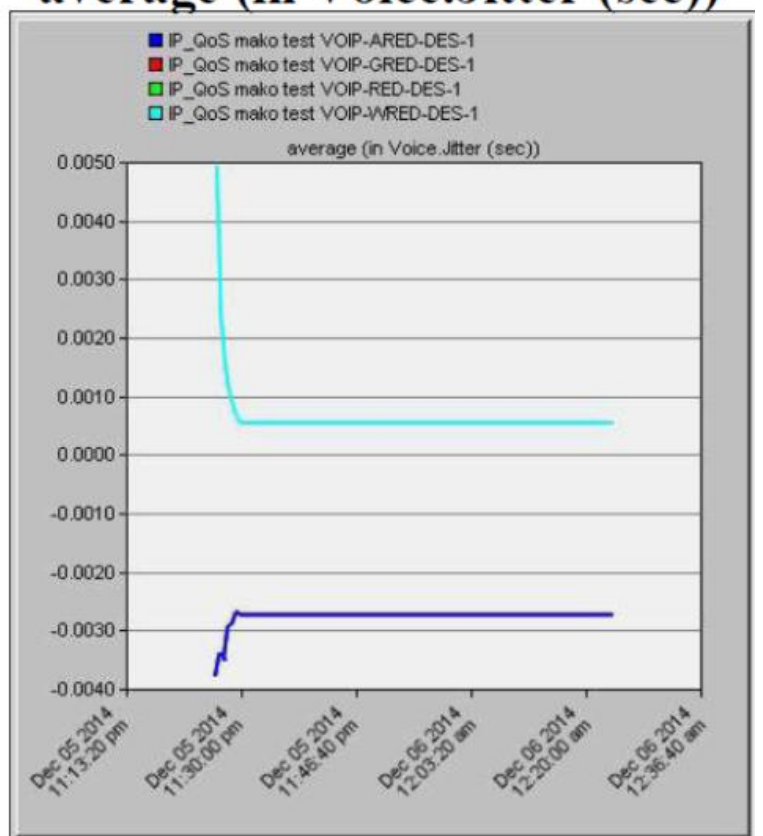

Figure 6.10: shows the graphic result of average jitter average (in Voice.Packet End-to-End Delay (sec))

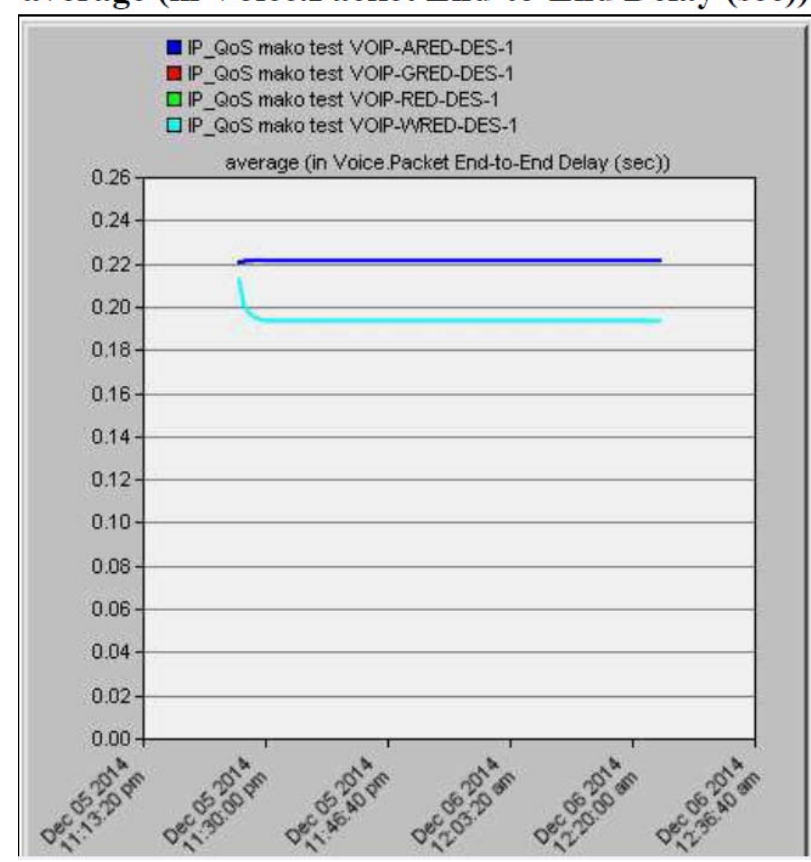

Figure 6.11: shows the graphic result of voice packet $\mathrm{E}$ to E delay

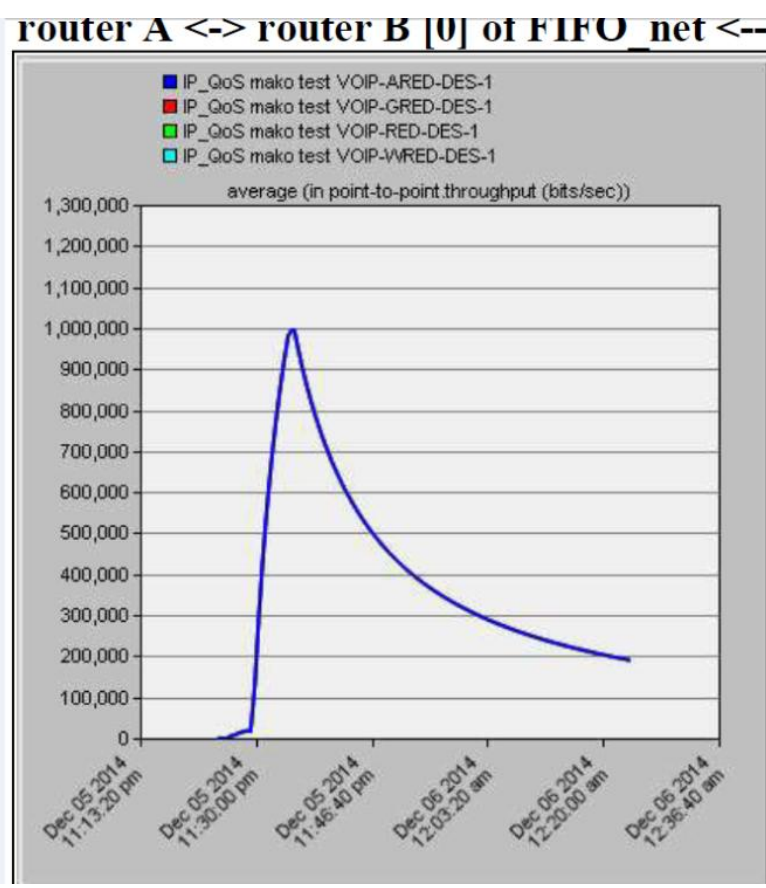

Figure 6.12: shows the graphic result Router A to B FIFO

\section{router $A$ of FIFO net}

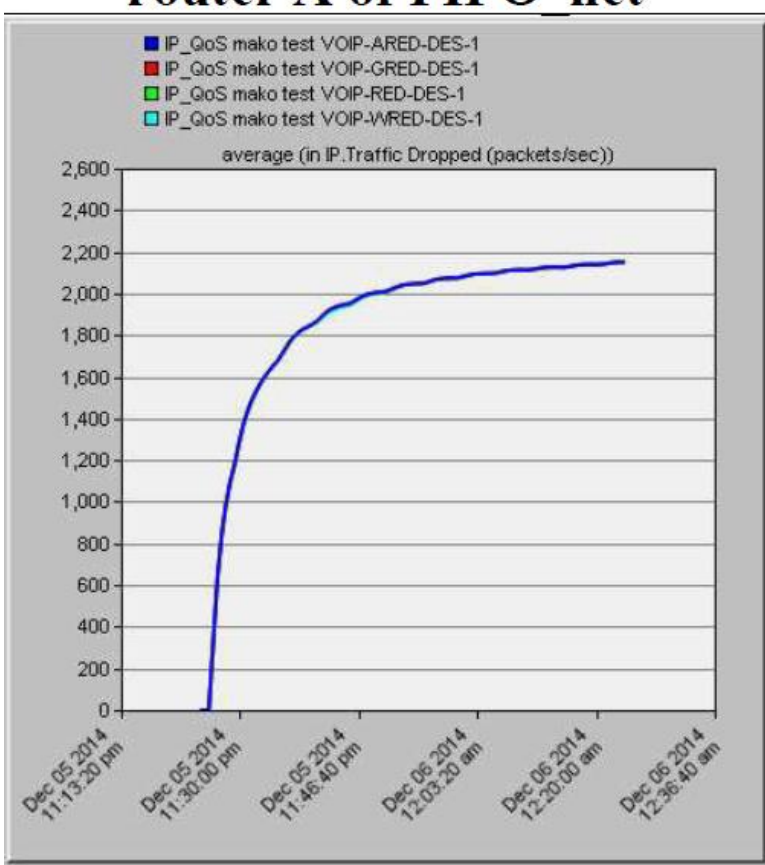

Figure 6.13: shows the graphic result Router A FIFO

When the number of users is increased to 300 user we can see that WRED start with a high value of jitter and then suddenly this value decrease also, we can notice that ARED shows the less jitter value among the other mechanism, In figure (d) ARED show the less traffic dropped while the other mechanism shows more .

\section{Conclusions}

This study evaluated the performance of VoIP over Ethernet WAN by applying AQM mechanism OPNET simulation tool. A comparison has been conducted

Volume 5 Issue 2, February 2016 


\section{International Journal of Science and Research (IJSR) \\ ISSN (Online): 2319-7064}

Index Copernicus Value (2013): 6.14 | Impact Factor (2013): 4.438

between four of (AQM Active Queue Management) methods RED, ARED, GRED and WRED This comparison aimed to identify which method offers more satisfactory performance measures for application like VOIP. A decision which method offers more satisfactory performance measure results is only made depending on varying the number of users and number of maximum queue size in the routers, it has been found that the configuration of the RED algorithm and GRED is not axiomatic. They must consider many situations such as network traffic, the traffic type, nature and duration of possible bursts, delays in the network, etc. It has been seen that in general, the ARED and GRED algorithms performs better, achieving a lower discard rate and lower overall delay. One problem that has been is the average queue size parameter tends to oscillate, especially given configuration changes and wq the minimum threshold, so care must be taken to configure. In general, in addition to everything said in this section, it has been shown that OPNET is a powerful tool and can be extremely useful for modeling and computer networks communication environments professionally. It can be very useful also at academics, to illustrate issues and network protocols, because of their axiomatic GUI, whether they have been previously described steps to follow through a tutorial style to what has been done here.

\section{Recommendations}

This study used the comparison according to one type of traffic, it recommend that to use some other traffic such as Http or video to see how this mechanism will deal with the VoIP packets while there is another types of packet also others parameters could be considered such as different encoder scheme and number of voice frame per packet.

\section{References}

[1] A Comparison of Active Queue Management Algorithms Using the OPNET Modeler"Chengyu Zhu and Oliver W. W. Yang, University of Ottawa James Aweya, Michel Ouellette, and Delfin Y. Montuno, Nortel Networks

[2] Taxonomy of Active Queue Management Strategies in Context of Peer-to-Peer Scenarios"KálmánGraffi, Konstantin Pussep, Nicolas Liebau, Ralf Steinmetz

[3] Floyd, Sally; Jacobson, Van (August 1993). "Random Early Detection (RED) gateways for Congestion Avoidance

[4] ACTIVE QUEUE MANAGEMENT ALGORITHMS FOR DOCSIS 3.0A Simulation Study of CoDel, SFQCoDeland PIE in DOCSIS 3.0 Networks, Greg White 2013

[5] Analysis of Active Queue Management Jae Chung and Mark ClaypoolComputer Science Department Worcester Polytechnic Institute Worcester, MA 01609, USA,

[6] S. Floyd and V. Jacobson. Random Early Detection Gatewaysfor Congestion Avoidance. IEEE/ACM Transactions on Networking, Aug. 1993.
[7] M. Christiansen, K. Jeffay, D. Ott, and F. Smith. Tuning RED for Web Traffic. In Proceedings of ACM SIGCOMM,Aug. 2000.

[8] C. Diot, G.Iannaccone, and M. May. Aggregate Traffic Performance with Active Queue Management and Drop from Tail. Sprint ATL Technical Report TR01-ATL012501, 2001.

[9] The RED Algorithm - Averaged Queue Weight Modeling for Non Linear Traffic Sridhar Madipelli , David Raj Gillella ,Sudhakar Devaraya Blekinge Institute of Technology 2009

[10] Sally Floyd, Ramakrishna Gummadi, , and Scott Shenker. Adaptive red: An algorithm for increasing the robustness of red's active queue management.

Technical report, 2001

[11] [16] Performance Studies of VoIP over Ethernet LANs Di Wu School of Computing and Mathematical Sciences, 2008

[12] Floyd, Sally; Jacobson, Van (August 1993). "Random Early Detection (RED)gateways for Congestion Avoidance A Comparison of Active Queue Management Algorithms Using the OPNET Modeler"Chengyu Zhu and Oliver W. W. Yang, University of Ottawa James Aweya, Michel Ouellette, and Delfin Y. Montuno, Nortel Networks

[13] [Aweya J., Ouellette M., and Montuno D.Y. "A Control Theoretic Approach To Active Queue Management," Computer International Journal on New Computer Architectures and Their Applications (IJNCAA) 2(1): 286-301 The Society of Digital Information and Wireless Communications, 2012 (ISSN: 2220-9085) 299 Networks, volume 36, issue 23 , pp. 203-

[14] [Floyd S., Ramakrishnan G., and Shenker S. "Adaptive RED: An Algorithm For Increasing the Robustness of RED's Active Queue Management," Technical report, ICSI, August 1, 2001. 